\title{
Diversity of the Wintertime Arctic Oscillation Pattern among CMIP5 Models: Role of the Stratospheric Polar Vortex
}

\author{
Hainan Gong, Lin WANG, AND Wen CHEN \\ Center for Monsoon System Research, Institute of Atmospheric Physics, Chinese Academy of Sciences, and College of \\ Earth and Planetary Sciences, University of Chinese Academy of Sciences, Beijing, China \\ RENGUANG WU \\ Center for Monsoon System Research, Institute of Atmospheric Physics, Chinese Academy of Sciences, Beijing, \\ and School of Earth Sciences, Zhejiang University, Hangzhou, Zhejiang, China \\ WEN ZHOU \\ Guy Carpenter Asia-Pacific Climate Impact Centre, School of Energy and Environment, City University \\ of Hong Kong, Hong Kong, China \\ LIN LIU \\ State Key Laboratory of Severe Weather, Chinese Academy of Meteorological Sciences, Beijing, China \\ DEBASHIS NATH AND XIAOQING LAN \\ Center for Monsoon System Research, Institute of Atmospheric Physics, Chinese Academy of Sciences, Beijing, China
}

(Manuscript received 12 September 2018, in final form 16 May 2019)

\begin{abstract}
The wintertime Arctic Oscillation (AO) pattern in phase 5 of the Coupled Model Intercomparison Project (CMIP5) climate models displays notable differences from the reanalysis. The North Pacific center of the AO pattern is larger in the ensemble mean of 27 models than in the reanalysis, and the magnitude of the North Pacific center of the AO pattern varies largely among the models. This study investigates the plausible sources of the diversity of the AO pattern in the models. Analysis indicates that the amplitude of the North Pacific center is associated with the coupling between the North Pacific and North Atlantic, which in turn is primarily modulated by the strength of the stratospheric polar vortex. A comparative analysis is conducted for the strong polar vortex (SPV) and weak polar vortex (WPV) models. It reveals that a stronger stratospheric polar vortex induces more planetary waves to reflect from the North Pacific to the North Atlantic and more wave activity fluxes to propagate from the North Pacific to the North Atlantic in the SPV models than in the WPV models. Thus, the coupling of atmospheric circulation between the North Pacific and North Atlantic is stronger in the SPV models, which facilitates more North Pacific variability to be involved in the AO variability and induces a stronger North Pacific center in the AO pattern. The increase in vertical resolution may improve the simulation of the stratospheric polar vortex and thereby reduces the model biases in the North Pacific-North Atlantic coupling and thereby the amplitude of the North Pacific center of the AO pattern in models.
\end{abstract}

\section{Introduction}

The Arctic Oscillation (AO) is the most dominant low-frequency mode of atmospheric variability in the extratropical Northern Hemisphere (Thompson and

Corresponding author: Dr. Lin Wang, wanglin@mail.iap.ac.cn
Wallace 1998). It is also known as the northern annular mode because of its annular structure in the sea level pressure (SLP) field (Thompson and Wallace 2000). Meanwhile, since the North Atlantic Oscillation (NAO; Hurrell 1995) index has high consistency with the AO index, the AO and NAO are generally accepted as the same phenomenon with different paradigms reported in 
many previous studies (e.g., Deser 2000; Wallace 2000; Rogers and McHugh 2002; Feldstein and Franzke 2006; Gong et al. 2017, 2018). The mass redistributions associated with different phases of the AO adjust the meridional gradient of the atmospheric pressure and significantly influence the weather and climate in the Northern Hemisphere (e.g., Thompson and Wallace 2000; Tan et al. 2008; Wang and Chen 2010; Chen et al. 2013). Also, the AO has significant implications for climate change in the Northern Hemisphere (Thompson et al. 2000). However, since the AO was proposed, the physical significance of the mode has been a matter of great debate (e.g., Ambaum et al. 2001; Honda and Nakamura 2001; Dommenget and Latif 2002; Wallace and Thompson 2002). Ambaum et al. (2001) considered a three-point seesaw system representing three centers of action: the Arctic, Euro-Atlantic, and Pacific regions. They suspected that the North Pacific variability, together with the NAO, constitutes the AO and that the Pacific center of the AO may develop from the North Pacific variability. Honda and Nakamura (2001) inferred that the leading mode of the SLP variability during the entire cold season might be interpreted as a superposition of the Aleutian low and Iceland low (AL-IL) seesaw on a dominant signal of the Arcticmidlatitude dipole. Besides, Dommenget and Latif (2002) suggested that one should be very careful in interpreting the EOF or rotated EOF modes as the potential physical modes. Wallace and Thompson (2002) explained that the correlation between the Pacific and Atlantic centers becomes stronger when the second EOF mode of the SLP is linearly removed from the original SLP field and that AO is a reliable physical mode. The discussion about the nature of the AO continues in recent years (e.g., Itoh 2008; Sun and Tan 2013; Gong et al. 2018).

Climate models are essential tools for understanding past climate variability and making projections of future climate change. It is particularly crucial for climate models to reproduce a realistic AO pattern. Previous studies indicated that the observed AO pattern could be roughly captured in most of the climate models, but the intermodel spread of the simulated AO pattern is large (e.g., Zuo et al. 2013; Gong et al. 2017). Meanwhile, the AO pattern generally shows a stronger Pacific center and weaker Atlantic center in many climate models compared to that in observations (Miller et al. 2006; Stoner et al. 2009; Cattiaux and Cassou 2013; Zuo et al. 2013; Gong et al. 2017). Gong et al. (2017) indicated that an excessively strong North Pacific center of the AO pattern is closely tied to a stronger relationship between the NAO and regional mode over the North Pacific [North Pacific mode (NPM)] in the models. In other words, some of North Pacific variability is involved in the AO patterns in the models. When the North Pacific variability is linearly removed from the $\mathrm{AO}$, the reproducibility of the AO pattern has improved significantly in the models. Based on the observational analysis, Gong et al. (2018) further demonstrated that the intensity of the North Pacific center of the AO pattern in observations is largely modulated by the coupling of atmospheric circulation over the North Pacific and the North Atlantic. When the North Pacific-North Atlantic coupling strengthens, the amplitude of the North Pacific center is stronger. The North Pacific-North Atlantic coupling can contribute more than $80 \%$ of the variability of the $\mathrm{AO}$ pattern during the past several decades.

Although the North Pacific-North Atlantic coupling is an important factor modulating the observed amplitude of the North Pacific center in the AO pattern, some questions remain unresolved. For example, whether this coupling in climate models essentially influences the amplitudes of the North Pacific center of the AO pattern? What physical processes are responsible for the North Pacific-North Atlantic coupling in climate models? Moreover, it is not clear whether other factors can also contribute to the diversity of the AO pattern, especially for the North Pacific and North Atlantic centers in models. The above questions will be addressed in this study in detail. The rest of the paper is organized as follows. Section 2 describes the data and methods. Section 3 investigates the possible factors and related physical processes responsible for the diversity of the wintertime AO pattern, especially for the North Pacific and North Atlantic centers in climate models. A summary and discussion are presented in section 4 .

\section{Data and methods}

\section{a. Data}

In this study, the observational proxies of the atmospheric variables are from the monthly mean data of the Japanese 55-yr Reanalysis (JRA-55) that span the period from January 1958 to the present with a horizontal resolution of $1.25^{\circ} \times 1.25^{\circ}($ Kobayashi et al. 2015 ; Harada et al. 2016). The outputs of the historical experiments during the period of 1961-2005 are employed from 27 models from phase 5 of the Coupled Model Intercomparison Project (CMIP5) (Table 1). Since the number of available ensemble members differs among individual models and these members are generally clustered to each other to compare the intermodel spread, we consider only the first realization from each CMIP5 model to maintain equal weighting across the models. To facilitate the comparison with reanalysis and 
TABLE 1. The climate modeling centers and CMIP5 models used in this study. The horizontal resolution and vertical levels (denoted by L) of each model are also listed.

\begin{tabular}{|c|c|c|}
\hline Model & Center & Atmospheric resolution \\
\hline ACCESS1.3 & $\begin{array}{l}\text { Commonwealth Scientific and Industrial Research Organization (CSIRO) } \\
\text { and Bureau of Meteorology (BOM), Australia }\end{array}$ & $192 \times 144$, L38 \\
\hline BCC_CSM1.1 & Beijing Climate Center, China Meteorological Administration & $128 \times 64, \mathrm{~L} 26$ \\
\hline BCC_CSM1.1(m) & Beijing Climate Center, China Meteorological Administration & $320 \times 160, \mathrm{~L} 26$ \\
\hline BNU-ESM & $\begin{array}{l}\text { College of Global Change and Earth System Science, Beijing Normal } \\
\text { University }\end{array}$ & $128 \times 64, \mathrm{~L} 26$ \\
\hline CESM1(CAM5) & Community Earth System Model Contributors & $288 \times 192, \mathrm{~L} 30$ \\
\hline CESM1(FASTCHEM) & Community Earth System Model Contributors & $288 \times 192, \mathrm{~L} 26$ \\
\hline CMCC-CM & Centro Euro-Mediterraneo per I Cambiamenti Climatici & $480 \times 240, \mathrm{~L} 31$ \\
\hline CNRM-CM5 & $\begin{array}{l}\text { Centre National de Recherches Météorologiques/Centre Européen de } \\
\text { Recherche et Formation Avancée en Calcul Scientifique }\end{array}$ & $256 \times 128, \mathrm{~L} 31$ \\
\hline FGOALS-g2 & $\begin{array}{l}\text { LASG, Institute of Atmospheric Physics, Chinese Academy of Sciences and } \\
\text { CESS, Tsinghua University }\end{array}$ & $128 \times 60, \mathrm{~L} 26$ \\
\hline GFDL CM2.1 & NOAA Geophysical Fluid Dynamics Laboratory & $144 \times 90, \mathrm{~L} 24$ \\
\hline GFDL CM3 & NOAA Geophysical Fluid Dynamics Laboratory & $144 \times 90, \mathrm{~L} 48$ \\
\hline GFDL-ESM2G & NOAA Geophysical Fluid Dynamics Laboratory & $144 \times 90, \mathrm{~L} 24$ \\
\hline GISS-E2-H & NASA Goddard Institute for Space Studies & $144 \times 90, \mathrm{~L} 40$ \\
\hline GISS-E2-R & NASA Goddard Institute for Space Studies & $144 \times 90, \mathrm{~L} 40$ \\
\hline HadCM3 & $\begin{array}{l}\text { Met Office Hadley Centre (additional HadGEM2-ES realizations } \\
\text { contributed by Instituto Nacional de Pesquisas Espaciais) }\end{array}$ & $96 \times 73$, L19 \\
\hline HadGEM2-AO & $\begin{array}{l}\text { National Institute of Meteorological Research/Korea Meteorological } \\
\text { Administration }\end{array}$ & $192 \times 144$, L38 \\
\hline HadGEM2-ES & $\begin{array}{l}\text { Met Office Hadley Centre (additional HadGEM2-ES realizations } \\
\text { contributed by Instituto Nacional de Pesquisas Espaciais) }\end{array}$ & $192 \times 144$, L38 \\
\hline INM-CM4.0 & Institute for Numerical Mathematics & $180 \times 120, \mathrm{~L} 21$ \\
\hline IPSL-CM5A-MR & Institute Pierre-Simon Laplace & $144 \times 143$, L39 \\
\hline IPSL-CM5B-LR & Institute Pierre-Simon Laplace & $96 \times 96$, L39 \\
\hline MIROC4h & $\begin{array}{l}\text { Atmosphere and Ocean Research Institute (The University of Tokyo), } \\
\text { National Institute for Environmental Studies, and Japan Agency for } \\
\text { Marine-Earth Science and Technology }\end{array}$ & $640 \times 320$, L56 \\
\hline MIROC-ESM & $\begin{array}{l}\text { Japan Agency for Marine-Earth Science and Technology, Atmosphere and } \\
\text { Ocean Research Institute (The University of Tokyo), and National } \\
\text { Institute for Environmental Studies }\end{array}$ & $128 \times 64, \mathrm{~L} 80$ \\
\hline MIROC-ESM-CHEM & $\begin{array}{l}\text { Japan Agency for Marine-Earth Science and Technology, Atmosphere and } \\
\text { Ocean Research Institute (The University of Tokyo), and National } \\
\text { Institute for Environmental Studies }\end{array}$ & $128 \times 64, \mathrm{~L} 80$ \\
\hline MPI-ESM-LR & $\begin{array}{l}\text { Max-Planck-Institut für Meteorologie (Max Planck Institute for } \\
\text { Meteorology) }\end{array}$ & $192 \times 96, \mathrm{~L} 47$ \\
\hline MPI-ESM-MR & $\begin{array}{l}\text { Max-Planck-Institut für Meteorologie (Max Planck Institute for } \\
\text { Meteorology) }\end{array}$ & $192 \times 96$, L95 \\
\hline MPI-ESM-P & $\begin{array}{l}\text { Max-Planck-Institut für Meteorologie (Max Planck Institute for } \\
\text { Meteorology) }\end{array}$ & $192 \times 96, \mathrm{~L} 47$ \\
\hline NorESM1-M & Norwegian Climate Centre & $144 \times 96, \mathrm{~L} 26$ \\
\hline
\end{tabular}

among other models, all the model data were interpolated into the $2.5^{\circ} \times 2.5^{\circ}$ longitude-latitude grid before analyses. In this study, the boreal winter (December, January, and February) is considered, and winter 1961 refers to the 1960/61 winter.

\section{b. Methods}

The AO pattern is defined as the first EOF mode of the winter-mean SLP variability in the extratropical Northern Hemisphere $\left(20^{\circ}-90^{\circ} \mathrm{N}\right)$, and the AO index is the corresponding first principal component (PC) (Thompson and
Wallace 1998). To measure the intensity of the North Atlantic and North Pacific centers in the AO pattern in each model and reanalysis, an AO-related North Atlantic center intensity index (NACI) and a North Pacific center intensity index (NPCI) are defined using the areaaveraged AO-related SLP anomalies over the North Atlantic region $\left(20^{\circ}-55^{\circ} \mathrm{N}, 60^{\circ} \mathrm{W}-30^{\circ} \mathrm{E}\right)$ and the North Pacific region $\left(25^{\circ}-60^{\circ} \mathrm{N}, 150^{\circ} \mathrm{E}-120^{\circ} \mathrm{W}\right)$, respectively, as in our previous studies (Gong et al. 2017, 2018). The AO-related SLP anomalies are derived by regressing the winter-mean SLP onto the winter-mean AO index. 
The standard deviation of year-to-year wintertime SLP variations is used to measure the intensity of the wintertime SLP variability in the Northern Hemisphere in reanalysis and each model as follows:

$$
\mathrm{SD}_{i}=\sqrt{\frac{1}{N} \sum_{j=1961}^{2005}\left(\mathrm{SLP}_{j}-\overline{\mathrm{SLP}}\right)^{2}}
$$

where $N$ is the number of winters during 1961-2005 and $\overline{\mathrm{SLP}}$ is the climatological-mean SLP during 1961-2005; $\mathrm{SD}_{i}$ is the standard deviation of winter-mean SLP in the grid $i$. It should be noted that in this study the standard deviation of SLP variations is calculated based on the total SLP variability instead of that linearly associated with AO variability. Similar to the NACI and NPCI, the area-averaged standard deviation of wintertime interannual SLP variations over the North Atlantic region $\left(20^{\circ}-55^{\circ} \mathrm{N}, 60^{\circ} \mathrm{W}-30^{\circ} \mathrm{E}\right)$ and the North Pacific region $\left(25^{\circ}-60^{\circ} \mathrm{N}, 150^{\circ} \mathrm{E}-120^{\circ} \mathrm{W}\right)$ is calculated to measure the intensities of wintertime SLP variability in the North Atlantic (NAVI) and North Pacific (NPVI) during 1961-2005, respectively.

To depict the strength of the North Pacific-North Atlantic coupling, the temporal correlation coefficient between the winter-mean North Pacific index (NPI) and North Atlantic index (NAI) during 1961-2005, denoted as $r_{\mathrm{NP}, \mathrm{NA}}$, is employed. Unlike the previous indices over the North Pacific and North Atlantic that are based on a specific pattern (e.g., AO pattern, SLP standard deviation pattern), the NPI and NAI are the time series calculated as an area-averaged SLP in North Pacific $\left(25^{\circ}-60^{\circ} \mathrm{N}, 150^{\circ} \mathrm{E}-120^{\circ} \mathrm{W}\right)$ and North Atlantic $\left(20^{\circ}-\right.$ $\left.55^{\circ} \mathrm{N}, 60^{\circ} \mathrm{W}-30^{\circ} \mathrm{E}\right)$, respectively. Following Castanheira and Graf (2003), the 50-hPa zonal-mean zonal winds along $65^{\circ} \mathrm{N}$, that is, $U_{50}$, are chosen to represent the strength of the stratospheric polar vortex (PV). The three-dimensional wave activity flux (Plumb 1985) is employed to delineate the propagation of the planetary waves.

The multimodel ensemble (MME) represents the averaged performance in individual models. In this study, we first calculate the statistics for each model and then average the results across models to obtain the MME as follows:

$$
\text { MME }=\frac{1}{M} \sum_{k=1}^{M}\left(\text { Statistics }_{k}\right),
$$

where $M$ is the number of models, Statistics ${ }_{k}$ is the statistics (e.g., standard deviation, AO pattern) in the model $k$. For example, the MME of the AO pattern is calculated as the equally weighted average of all models' corresponding AO patterns. Similarly, the MME of the standard deviation of winter-mean SLP variability is calculated as the multimodel average of standard deviations in individual models. Here, the averaged $t$ value among models is used to evaluate the significance level of the MME pattern based on a two-tailed Student's $t$ test, following Gong et al. $(2014,2015)$.

\section{Sources of the diversity of the wintertime AO pattern among CMIP5 models}

In this section, we first compare the AO pattern between MME and JRA-55 reanalysis as well as among the individual models. Then, we explore the plausible reasons for the difference in the AO pattern among the models, including the impact of the SLP variability and the North Pacific-North Atlantic coupling. Last, we investigate the role of the stratospheric $\mathrm{PV}$ in modulating the North Pacific-North Atlantic coupled variations.

\section{a. Diversity of the wintertime AO pattern in CMIP5 models}

There is a notable difference in the wintertime AO pattern between the JRA-55 dataset and MME of 27 CMIP5 models. Figure 1a displays the spatial pattern of the leading EOF mode of wintertime SLP anomalies in the JRA-55 dataset, which represents the AO pattern. The AO pattern is quite similar to the NAO pattern, with stronger North Atlantic center and weaker North Pacific center, which is consistent with previous studies (e.g., Deser 2000; Feldstein and Franzke 2006; Gong et al. 2017, 2018). These characteristics are also present in ERA and NCEP-NCAR datasets (not shown). In the following, we refer the results obtained in the JRA-55 dataset as "reanalysis." In MME, however, the pattern of AO shows large differences from reanalysis (Fig. 1b), with much stronger North Pacific center and weaker North Atlantic center than in reanalysis. This feature is seen in most of the individual models (not shown) (e.g., Zuo et al. 2013; Gong et al. 2017).

Individual models show substantial differences in amplitude of the North Atlantic and North Pacific centers in the AO pattern. Figures $1 \mathrm{~d}$ and $1 \mathrm{e}$ show the NACI and NPCI in reanalysis, MME, and 27 CMIP5 models, respectively. The NACI in reanalysis and MME are 1.77 and $1.07 \mathrm{hPa}$, respectively. The NPCI in reanalysis and MME are 0.41 and $1.27 \mathrm{hPa}$, respectively. There is a large spread in NACI among the models with the maximum NACI of $1.76 \mathrm{hPa}$ and the minimum $\mathrm{NACI}$ of $0.56 \mathrm{hPa}$ (Fig. 1d). The minimum NPCI is near zero, and the maximum NPCI reaches $2.24 \mathrm{hPa}$ (Fig. 1e). Thus, the difference of NPCI in the models is much more significant than that of NACI. The intermodel standard 

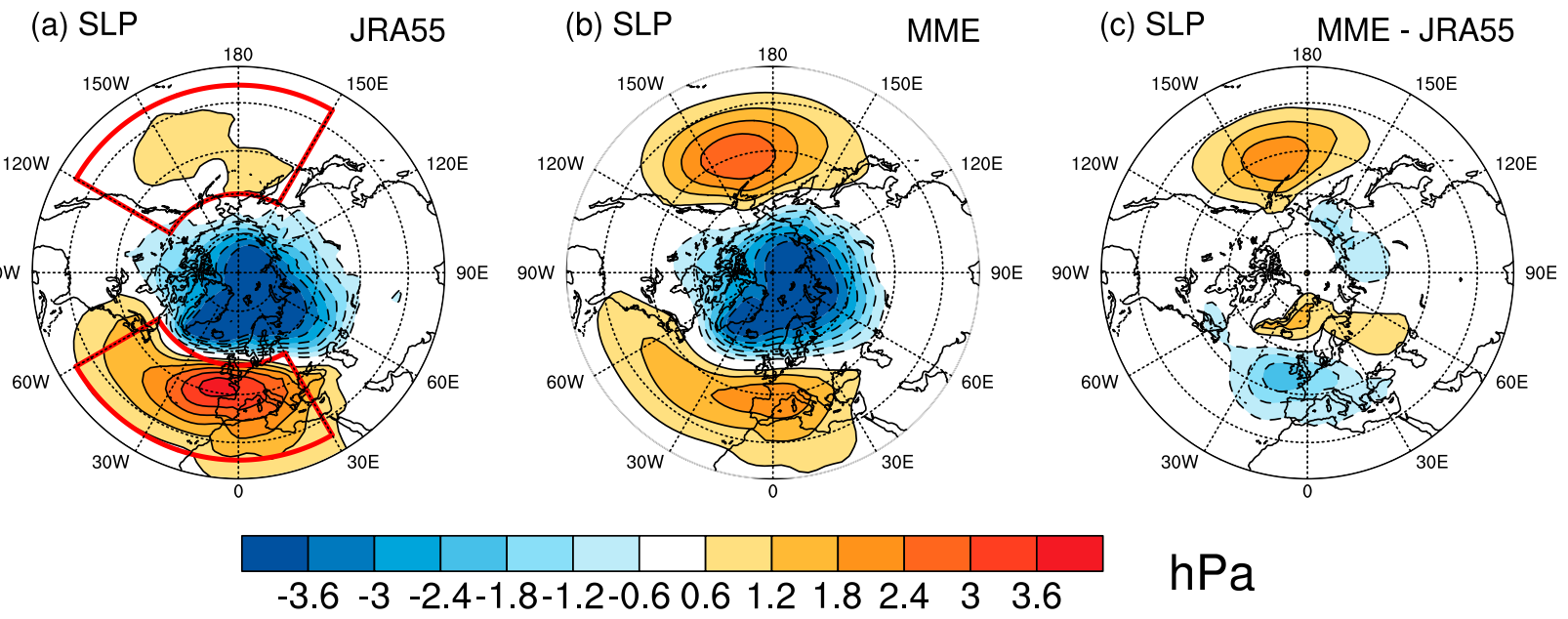

$\mathrm{hPa}$

(d) $\mathrm{NACl}$

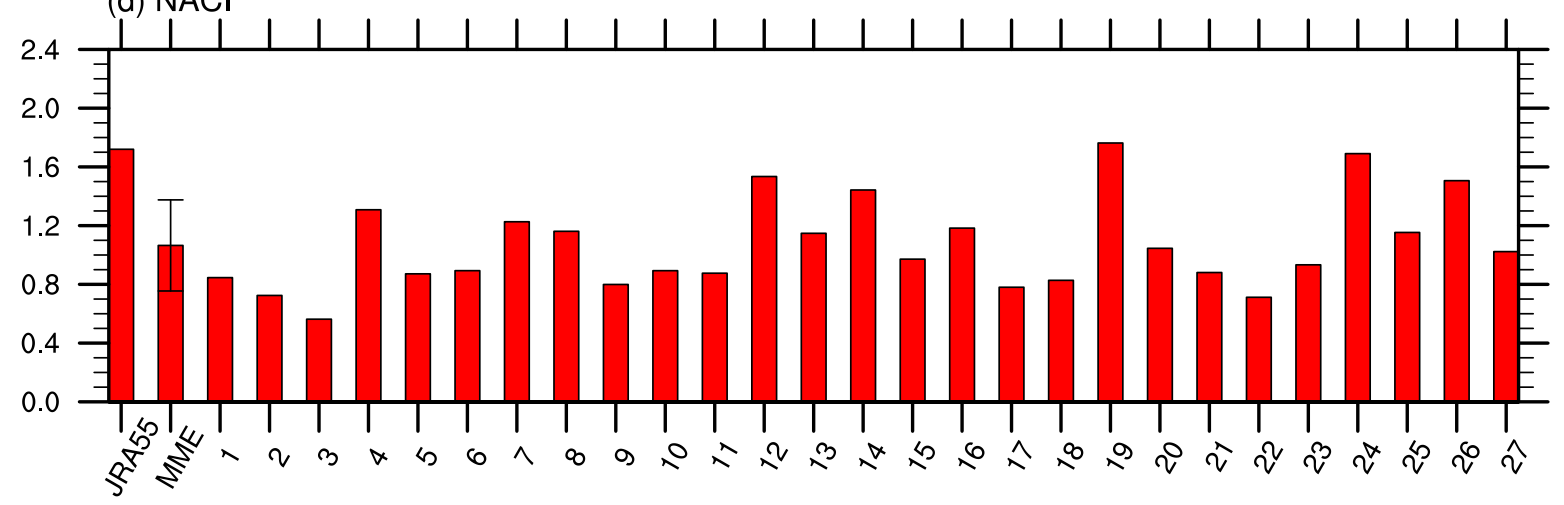

(e) NPCl

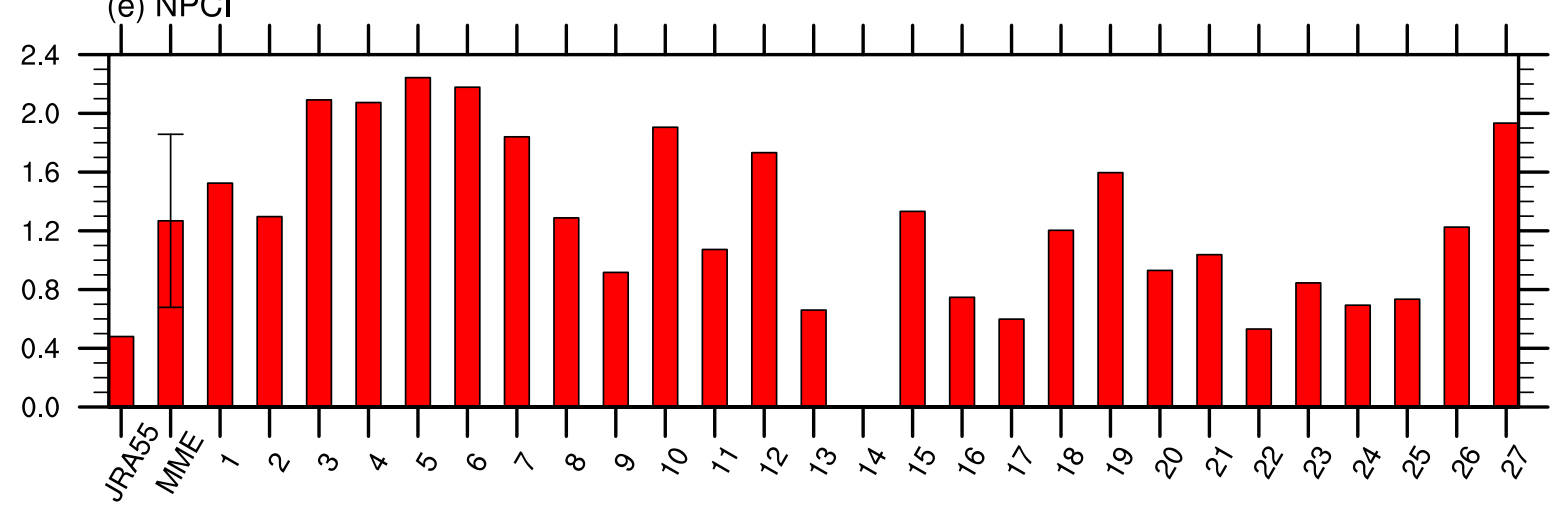

FIG. 1. The winter-mean SLP regressed on the normalized AO index in (a) reanalysis (JRA55) and (b) MME for the period of 19612005. (c) The difference between (b) and (a). (d) The intensity of the North Atlantic center of AO pattern (NACI) in reanalysis, MME, and 27 CMIP5 models, respectively. The error bar indicates one standard deviation of uncertainty. (e) As in (d), but for the intensity of North Pacific center of AO pattern (NPCI). The red boxes in (a) indicate the regions to define the indices in North Pacific and North Atlantic centers.

deviation of NPCI is almost twice than that of NACI. These results suggest that there is a vast diversity in $\mathrm{AO}$ pattern among the models, especially in its North Pacific center. This diversity largely influences the capacity of models in simulating the AO pattern realistically.
Hence, a question arises: what factors induce this diversity?

The AO is the dominant mode of SLP variability in the extratropical Northern Hemisphere. Thus, the different amplitudes of the centers in the wintertime AO 

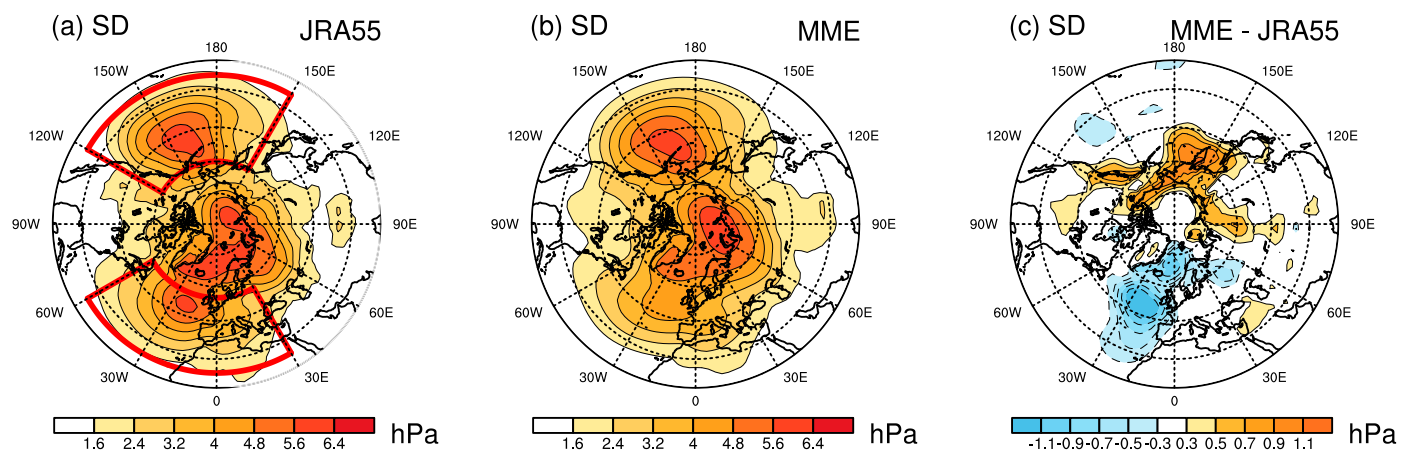

(d) $r=0.8$

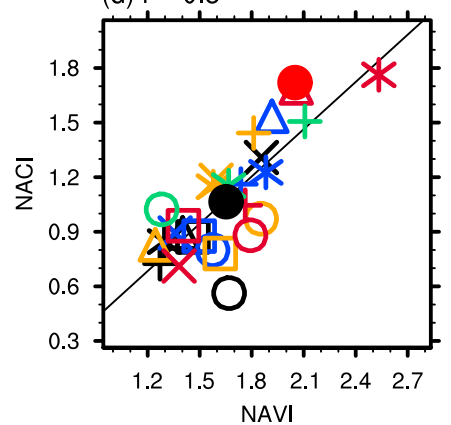

(e) $r=0.87$

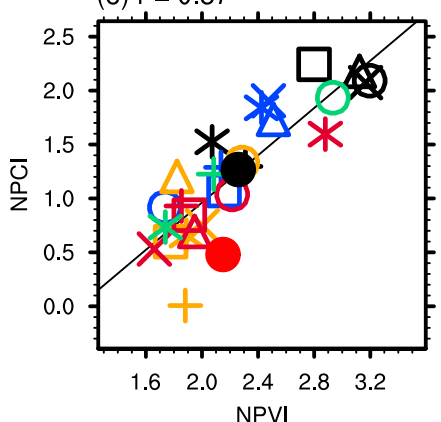

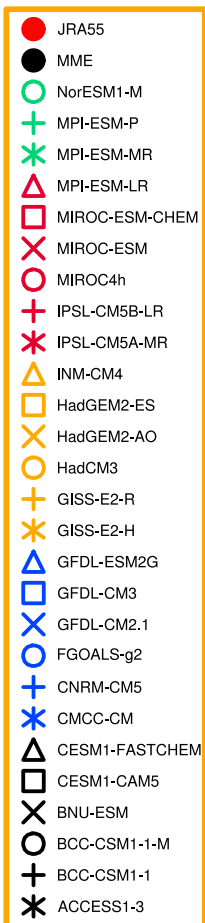

FIG. 2. The standard deviation of winter-mean SLP variability in the extratropical Northern Hemisphere in (a) reanalysis and (b) MME for the period 1961-2005. (c) The difference between (b) and (a). (d) Scatterplot of the NACI ( $y$ axis) vs the NAVI ( $x$ axis) in 27 CMIP5 models, MME, and reanalysis. The intermodel correlation coefficient $r$ between $x$ - and $y$-axis variables is also shown. (e) As in (d), but for the scatterplot of the NPCI ( $y$ axis) vs the NPVI ( $x$ axis).

pattern may be associated with different magnitudes of wintertime SLP variability. Moreover, previous studies indicated that the coupling of atmospheric circulation between the North Pacific and North Atlantic could modulate the intensity of the North Pacific center of the AO pattern in reanalysis (Gong et al. 2018). Therefore, we examine the possible roles of the above two factors in different amplitudes of the AO pattern in the following subsections.

\section{b. Role of the intensity of SLP variability and the North Pacific-North Atlantic coupling}

First, the possible association between the magnitude of SLP variability and the amplitudes of the North Pacific and North Atlantic centers is investigated. To illustrate this association, we show the observed and simulated magnitude of wintertime SLP variability over the Northern Hemisphere in Figs. 2a-c. Indeed, large SLP variability is located near the action centers of the AO pattern both in reanalysis and MME except for the North Pacific one in reanalysis (Figs. 2a,b and Figs. 1a,b). The SLP variability is large in the North Pacific, but the North Pacific center of the AO pattern is weak in reanalysis (Figs. 1a, 2a). The differences in the wintertime SLP variability between the MME and reanalysis display a distribution similar to that of the AO pattern, with weaker SLP variability in the North Atlantic and stronger SLP variability in the North Pacific (Figs. 1c, 2c). This result suggests that the amplitudes of the AO pattern may associate with the magnitude of SLP variability in the extratropical Northern Hemisphere.

To further demonstrate the association between wintertime SLP variability and the amplitude of the centers of the AO pattern in models, we compare the two quantities across the models. Figure $2 d$ shows the scatter diagram of the NACI and NAVI in 27 CMIP5 models, MME, and reanalysis. Each symbol represents the value in the corresponding model and reanalysis. From Fig. 2d, all the models simulate a weaker NACI than that in reanalysis, which thereby induces a weaker North Atlantic center of the AO pattern in MME compared to the reanalysis (Fig. 1b). Meanwhile, the NAVI is also weaker in most of the models (25/27) than in reanalysis (Fig. 2d). A smaller NAVI tends to correspond to a weaker NACI in models. The intermodel correlation coefficient between NACI and NAVI reaches 0.8 , exceeding the significance level at $0.1 \%$. It suggests that the amplitude of the North Atlantic center in the AO is associated with the magnitude of the SLP 
(a) $r=0.72$

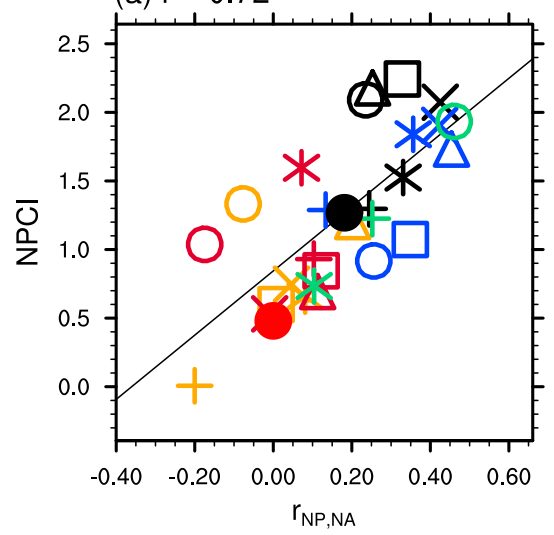

(b) SLP

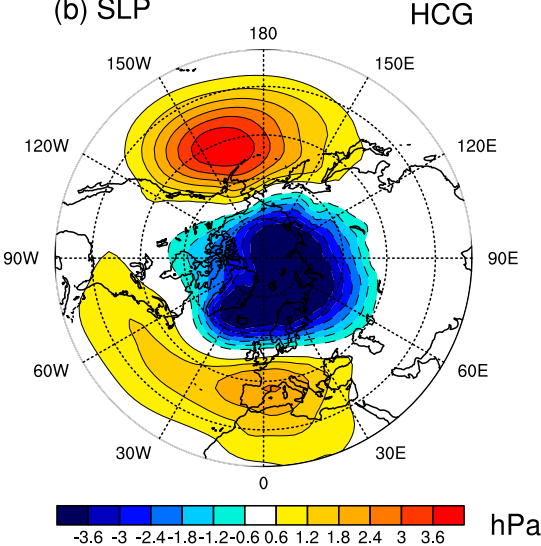

(c) SLP

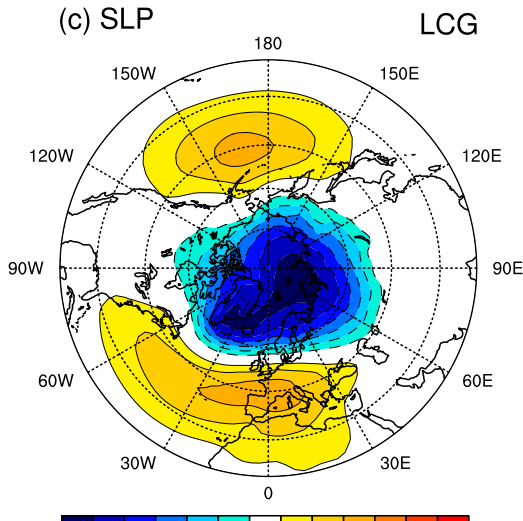

$3.6-3$-2.4-1.8-1.2-0.6 0.6121 .82433$.

\section{JRA55}

- MME

O NorESM1-M

+ MPI-ESM-P

* MPI-ESM-MR

$\triangle$ MPI-ESM-LR

$\square$ MIROC-ESM-CHEM

$\times$ MIROC-ESM

O MIROC4h

+ IPSL-CM5B-LR

* IPSL-CM5A-MR

$\triangle$ INM-CM4

7) HadGEM2-ES

X HadGEM2-AO

O HadCM 3

+ GISS-E2-R

* GISS-E2-H

$\triangle$ GFDL-ESM2G

$\square$ GFDL-CM3

$\times$ GFDL-CM2.1

O FGOALS-g2

+ CNRM-CM5

* CMCC-CM

$\triangle$ CESM1-FASTCHEM

$\square$ CESM1-CAM5

$\times$ BNU-ESM

O BCC-CSM1-1-M

+ BCC-CSM1-1

* ACCESS1-3

FIG. 3. (a) Scatterplot of the NPCI ( $y$ axis) vs $r_{\mathrm{NP}, \mathrm{NA}}(x$ axis) in 27 CMIP5 models, MME, and reanalysis. (b) The AO pattern of MME in eight high-correlation models (HCG). (c) As in (b), but for eight low correlation models (LCG).

variability in the North Atlantic, and the weaker North Atlantic center in the AO is related to the smaller SLP variability in models.

Similarly, Fig. 2e shows the scatter diagram of intermodel variability between the NPCI and NPVI in 27 CMIP5 models, MME, and reanalysis. The intermodel correlation coefficient between NPCI and NPVI reaches 0.87 , exceeding the significance level at $0.1 \%$. This result indicates that a larger NPVI tends to correspond to a stronger NPCI in models. However, it is noteworthy that almost all the models (26/27) show a stronger NPCI compared to the reanalysis, but only about half of the models (14/27) simulate a larger NPVI compared to reanalysis. The other models show a weaker NPVI than in reanalysis (Fig. 2e), and this inconsistency is also apparent in MME. Although the North Pacific center of the AO pattern is much stronger in MME than in reanalysis, the variability of SLP over the North Pacific is slightly larger in MME than in reanalysis. These results imply that there may be other factors modulating the amplitudes of North Pacific center of the AO pattern in models.

As mentioned above, the observed NPCI is very weak though the NPVI is large in reanalysis. Recent studies indicated that the AO in CMIP5 models is coupled with some North Pacific variability, and the North Pacific center of the AO pattern arises very likely from the coupling of the North Pacific and North Atlantic because AO is fundamentally rooted in the variability over the North Atlantic (Gong et al. 2017, 2018). Therefore, we further examine whether the amplitude of the North Pacific center of the AO pattern is essentially influenced by the North Pacific-North Atlantic coupling in the models.

Figure 3a shows the scatter diagram of the NPCI and $r_{\mathrm{NP}, \mathrm{NA}}$. The figure reveals that the observed $r_{\mathrm{NP}, \mathrm{NA}}$ is near zero, suggesting a fragile relationship between the SLP variations in the North Pacific and North Atlantic center in reanalysis. Most of the models (24/27) show a positive $r_{\mathrm{NP}, \mathrm{NA}}$, indicating a stronger North Pacific and 
North Atlantic coupling in the models than in reanalysis. This result is consistent with the fact that most of the models simulated a stronger North Pacific center of the AO pattern. Meanwhile, a larger $r_{\mathrm{NP}, \mathrm{NA}}$ tends to correspond to a more pronounced NPCI in the models. The intermodel correlation coefficient between them is 0.72 , exceeding the significance level at $0.1 \%$. Based on the $r_{\mathrm{NP}, \mathrm{NA}}$, the AO pattern of MME in eight highcorrelation models group (HCG) and eight lowcorrelation models group (LCG) are shown in Figs. $3 b$ and $3 c$. The North Pacific center of the AO pattern is much stronger in HCG models than in LCG models (Figs. 3d-f). Meanwhile, the intensity of the North Atlantic center is somewhat similar in the two groups (Figs. $3 \mathrm{~b}$ and $3 \mathrm{c}$ ). These results suggest that the amplitudes of the North Pacific center of the AO pattern are indeed related to the North Pacific-North Atlantic coupling in models. The positive value of $r_{\mathrm{NP}, \mathrm{NA}}$ in most of the models suggests a certain North Pacific-North Atlantic coupling. In this case, a larger North Pacific SLP variability may facilitate a stronger North Pacific center of the AO pattern in the models. Therefore, the high intermodel correlation between the NPCI and NPVI is not surprising (Fig. 2e). Since the AO variability is mainly rooted in the North Atlantic (e.g., Gong et al. 2018), the North Pacific-North Atlantic coupling and the amplitude of the North Pacific center reflect the degree of coherence between the North Pacific variations and the AO. Therefore, the coupling of the North Pacific and North Atlantic variations is an important factor for the amplitude of the North Pacific center in the AO. In this context, another question arises: what physical processes are responsible for the North PacificNorth Atlantic coupling in the models? It will be investigated in the following subsection.

\section{c. Modulation of the stratospheric PV on the North Pacific-North Atlantic coupling}

Previous studies have demonstrated that the coupling of atmospheric circulation between the two regions may be influenced by the tropospheric planetary wave reflection from one region to another (Castanheira and Graf 2003; Sun and Tan 2013; Yuan et al. 2018). Moreover, the vertical propagation, including upward and downward reflection of tropospheric planetary waves, mainly depends on the strength of the lower-stratospheric westerlies in winter (Charney and Drazin 1961). The tropospheric planetary waves could vertically propagate upward into the stratosphere when the velocity of zonalmean stratospheric westerly is weaker than the critical Rossby velocity. Otherwise, they will be trapped in the troposphere and alter the tropospheric circulations. This process may facilitate the establishment of coherent variations between the two regions. Therefore, the stratospheric PV, which represents the most dominant feature of the stratospheric circulation over the winter pole, may influence the coupling of tropospheric circulation through changing the propagation of planetary waves during boreal winter (e.g., Perlwitz and Graf 2001; Chen et al. 2002, 2003; Castanheira and Graf 2003; Sun and Tan 2013).

To examine the possible effects of the stratospheric PV on the North Pacific-North Atlantic coupling, we use the 50 -hPa zonal-mean zonal winds along $65^{\circ} \mathrm{N}$, that is, $U_{50}$, to represent the strength of the stratospheric PV (Castanheira and Graf 2003). Figures $4 \mathrm{a}$ and $4 \mathrm{~b}$ show the scatter diagram of $r_{\mathrm{NP}, \mathrm{NA}}$ and NPCI with climatologicalmean PV, respectively. Indeed, a stronger climatological stratospheric PV tends to correspond to a much tighter North Pacific-North Atlantic coupling as well as a stronger North Pacific center of the AO pattern in models. The intermodel correlation coefficients of $r_{\mathrm{NP}, \mathrm{NA}}$ and NPCI with climatological-mean stratospheric PV are 0.62 and 0.54 , respectively, both exceeding the significance level at $1 \%$. This result suggests that climatological-mean stratospheric PV in CMIP5 models may modulate the strength of the North PacificNorth Atlantic coupling as well as the intensity of North Pacific center of the AO pattern.

To confirm the above point and to interpret the mechanism of the stratospheric PV influence on the North Pacific-North Atlantic coupling, we divide the models into two groups (Table 2). The models whose climatological stratospheric $\mathrm{PV}$ is stronger than $18 \mathrm{~m} \mathrm{~s}^{-1}$ are classified into the strong polar vortex group (SPV), and the remaining models are classified into the weak polar vortex group (WPV). The above criterion was used in reanalysis by Sun and Tan (2013) for classifying the strong and weak stratospheric PV events. Note that the results of this study are not very sensitive to the choice of the threshold (not shown). Two-thirds of the models (18/27) are in the SPV and the rest of the models (9/27) are in the WPV group. It is also noted that all models in HCG are in SPV, and more than half the models (five out of eight) in LCG are in WPV. The North Pacific center of the AO pattern is much stronger in SPV models than in WPV models (Figs. 4c,d). Meanwhile, the intensity of the North Atlantic center is somewhat similar in these two groups (Figs. 4c,d). This result implies that the AO pattern in CMIP5 models indeed could be modulated by the stratospheric PV.

Figures 5a-c show the climatological wintertime zonal-mean zonal wind in SPV models, WPV models, and their differences. The zonal-mean zonal wind differences between SPV and WPV models exhibit a clear dipole in the upper troposphere to the stratosphere with 
(a) $r=0.62$

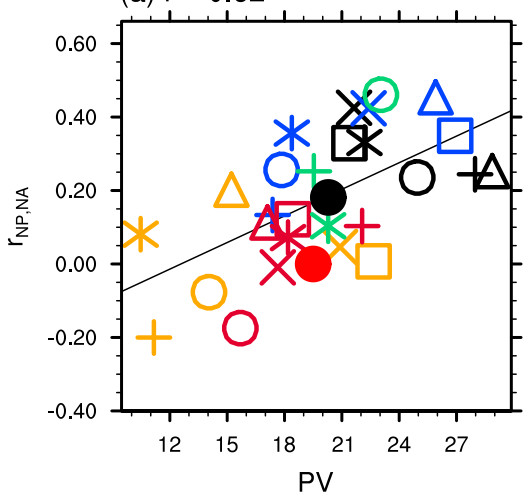

(c) SLP

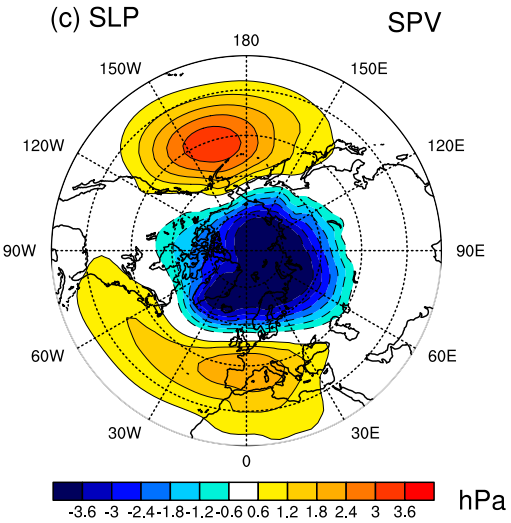

(b) $r=0.54$

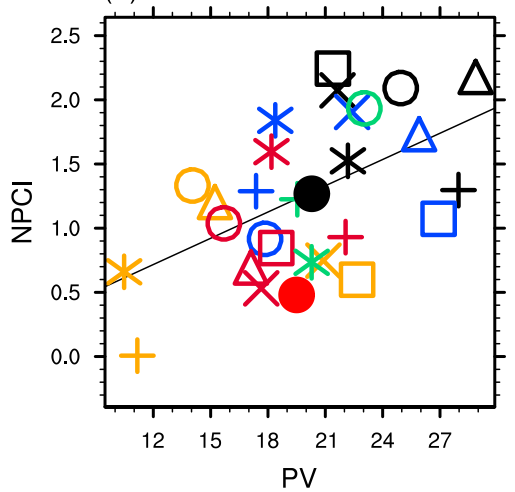

(d) SLP

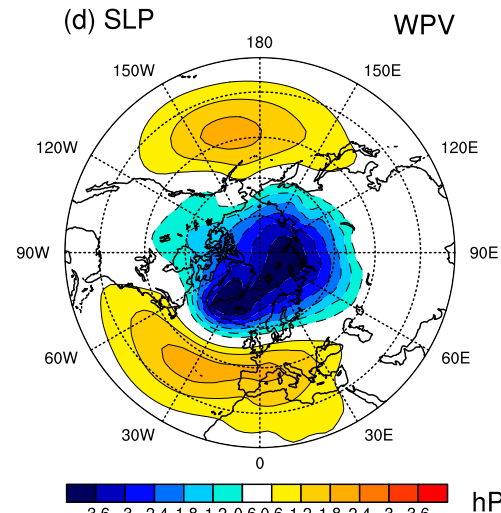

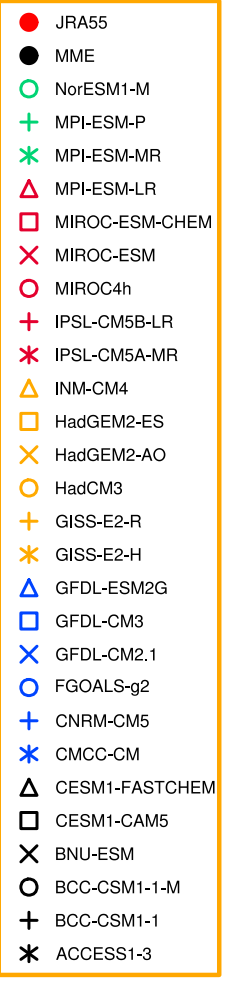

* ACCESS1-3

FIG. 4. (a) Scatterplot of the $r_{\text {NP,NA }}(y$ axis) vs climatological stratospheric polar vortex (PV; $x$ axis) in 27 CMIP5 models, MME, and reanalysis. (b) As in (a), but for the scatterplot of the NPCI ( $y$ axis) vs climatological stratospheric PV ( $x$ axis). (c) The AO pattern of MME in strong polar vortex models (SPV). (d) As in (c), but in weak polar vortex models (WPV).

the node located at $\sim 45^{\circ} \mathrm{N}$. As expected, the stratospheric polar jet stream is much stronger in SPV models than in WPV models (Fig. 5c). Figure 5d further displays the frequency distribution of monthly $50-\mathrm{hPa}$ zonalmean zonal wind at $65^{\circ} \mathrm{N}$ in SPV and WPV models during the winter. Larger wind speed is seen in SPV models. The wind speed with maximum frequency is about $25 \mathrm{~m} \mathrm{~s}^{-1}$ in SPV models, and it is only $16 \mathrm{~m} \mathrm{~s}^{-1}$ in WPV models. Meanwhile, there are more than threefourths of the months in which the wind speed is up to the standard $\left(18 \mathrm{~m} \mathrm{~s}^{-1}\right)$ in SPV models, while it is only one-third of the months in WPV models. These results indicate that there are more and stronger stratospheric PV events in SPV models than in WPV models. It is noteworthy that although the strength of the stratospheric PV events in WPV models is generally weak, there may exist some degree of North Pacific-North Atlantic coupling in WPV models though weaker (less frequent) than in SPV models. In other words, the modulation of climatological stratospheric PV on the strength of the North Pacific-North Atlantic coupling is mainly attributed to its control on the frequency of the strong North Pacific-North Atlantic coupling events.

To demonstrate the physical process responsible for the modulation of the stratospheric PV on the North Pacific-North Atlantic coupling, 100-hPa Northern Hemisphere planetary wave activity fluxes in SPV and WPV models during the winters of 1961-2005 and their differences are shown in Figs. 6a-c. The contours in Figs. $6 a-c$ indicate the vertical component of the wave activity fluxes, and the arrows are the horizontal

TABLE 2. List of the models grouped into the SPV and WPV.

\begin{tabular}{ll}
\hline \hline SPV & ACCESS1.3, BCC_CSM1.1, BCC_CSM1.1(m), \\
BNU-ESM, CESM1(CAM5), CESM1(FASTCHEM), \\
CMCC-CM, GFDL CM2.1, GFDL CM3, GFDL- \\
ESM2G, HadGEM2-AO, HadGEM2-ES, IPSL- \\
CM5A-MR, IPSL-CM5B-LR, MIROC-ESM-CHEM, \\
MPI-ESM-MR, MPI-ESM-P, NorESM1-M \\
CNRM-CM5, FGOALS-g2, GISS-E2-H, GISS-E2-R, \\
HadCM3, INM-CM4.0, MIROC4h, MIROC-ESM, \\
MPI-ESM-LR
\end{tabular}


(a) SPV

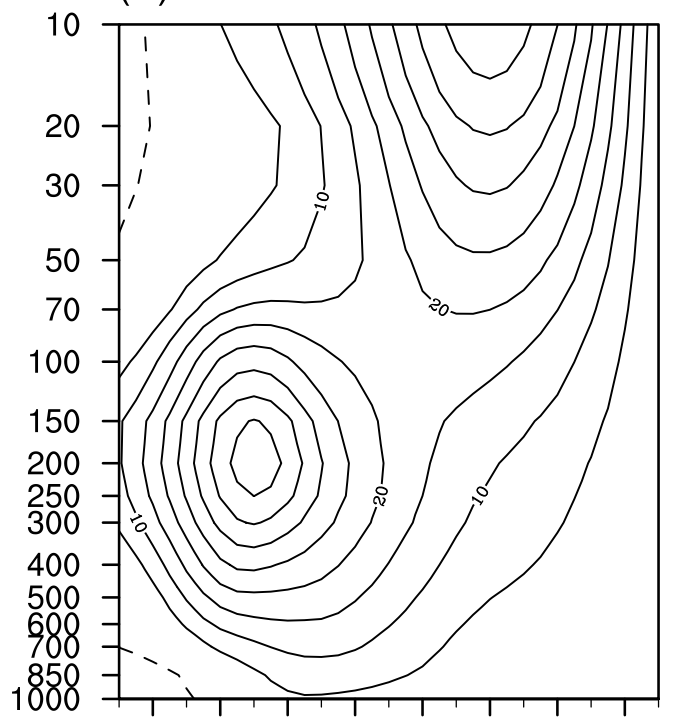

$15 N 25 N$ 35N 45N 55N 65N 75N 85N

(c) SPV - WPV

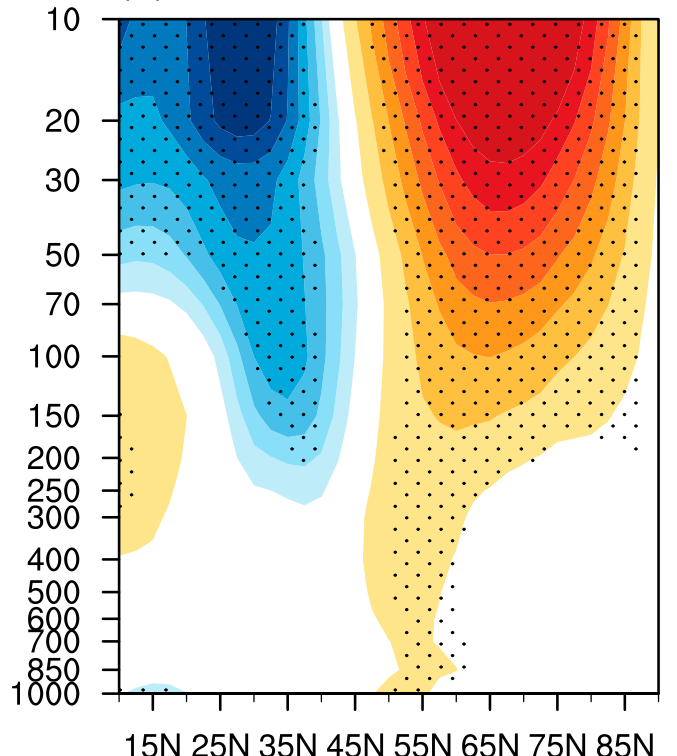

$\begin{array}{lllllllllllllll}-7 & -6 & -5 & -4 & -3 & -2 & -1 & 1 & 3 & 5 & 7 & 9 & 11 & 13\end{array}$ (b) WPV

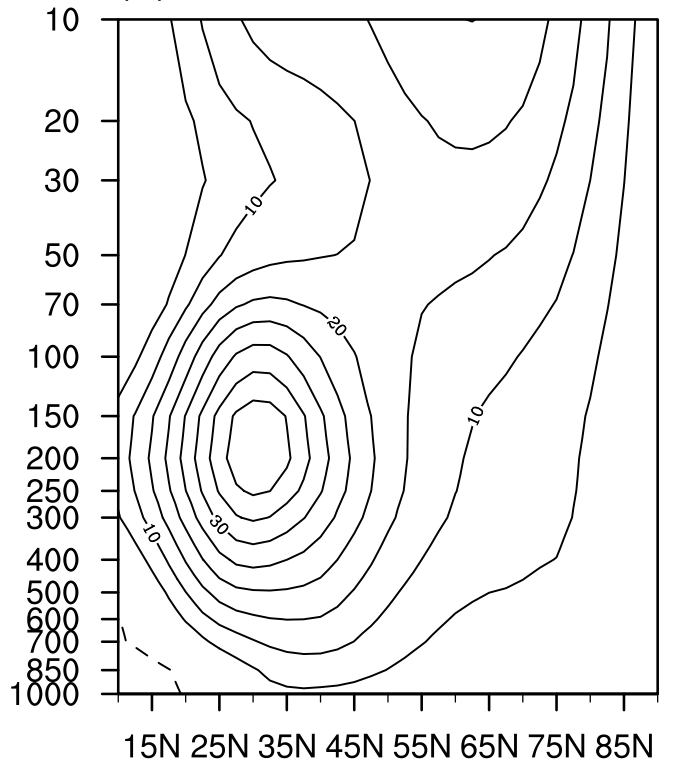

(d) $U_{65 \mathrm{~N}}$

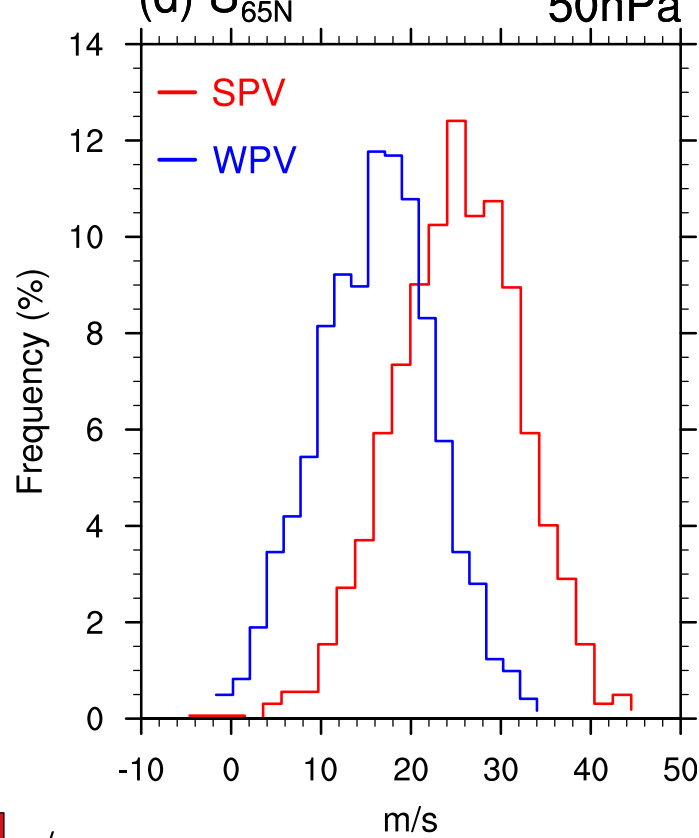

FIG. 5. Climatology of winter-mean (DJF) zonal-mean zonal wind in (a) SPV, (b) WPV, and (c) the differences between SPV and WPV. Contour intervals are $5 \mathrm{~m} \mathrm{~s}^{-1}$ in (a) and (b). (d) The frequency distribution of monthly 50$\mathrm{hPa}$ zonal-mean zonal wind at $65^{\circ} \mathrm{N}$ for SPV (red line) and WPV (blue line). Dots in (c) indicate the significance level at $5 \%$ based on a two-sided Student's $t$ test.

components. There are two distinct centers of upward wave activity fluxes over the eastern Asia-North Pacific and Europe regions. The upward wave activity fluxes are much stronger over eastern Asia-North Pacific than over Europe in both SPV and WPV models. There are apparent downward wave activity fluxes over the northern North America and North Atlantic in SPV models. The above evidence indicates that these upward-propagating planetary waves from East Asia-North Pacific are reflected by the strong stratospheric PV (Fig. 6a). The downward 
(a) SPV

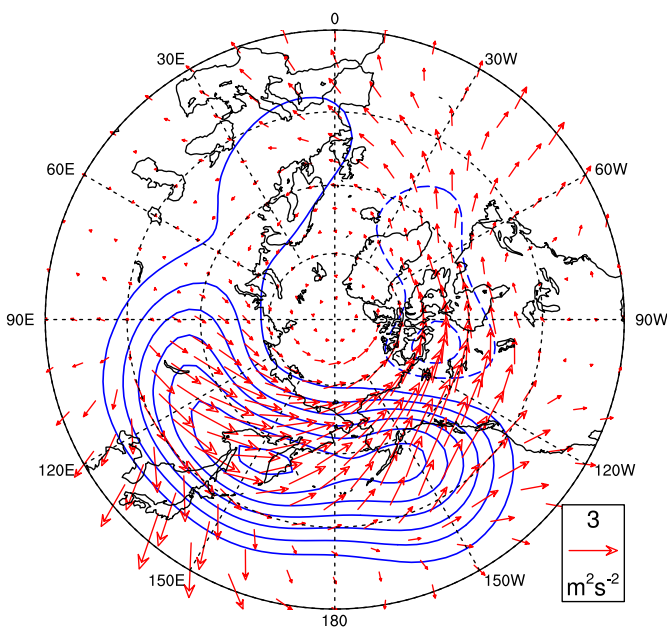

(b) WPV

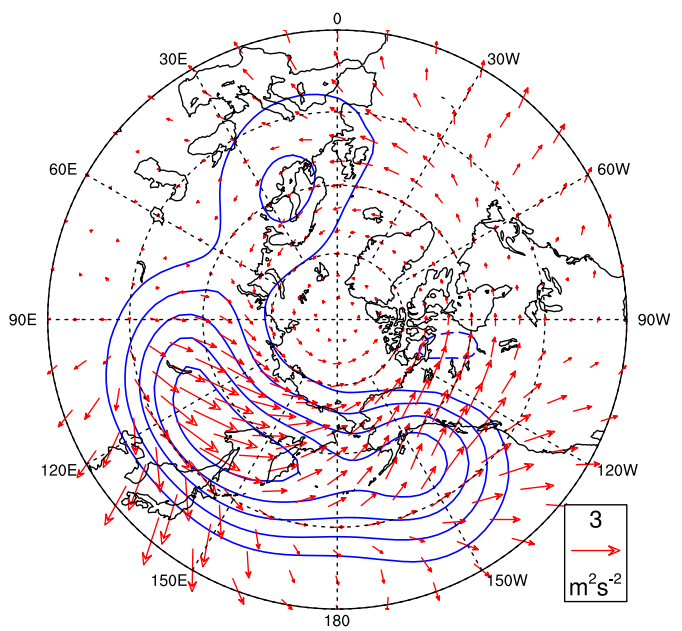

(c) SPV - WPV

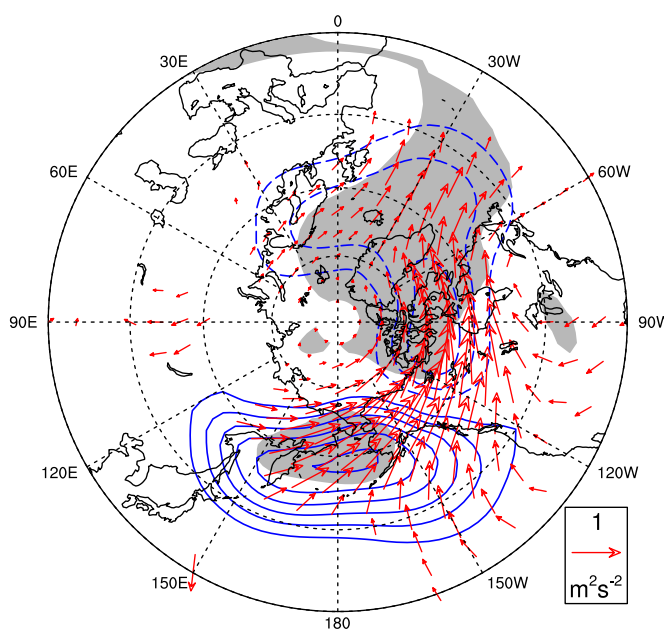

(d) SPV - WPV

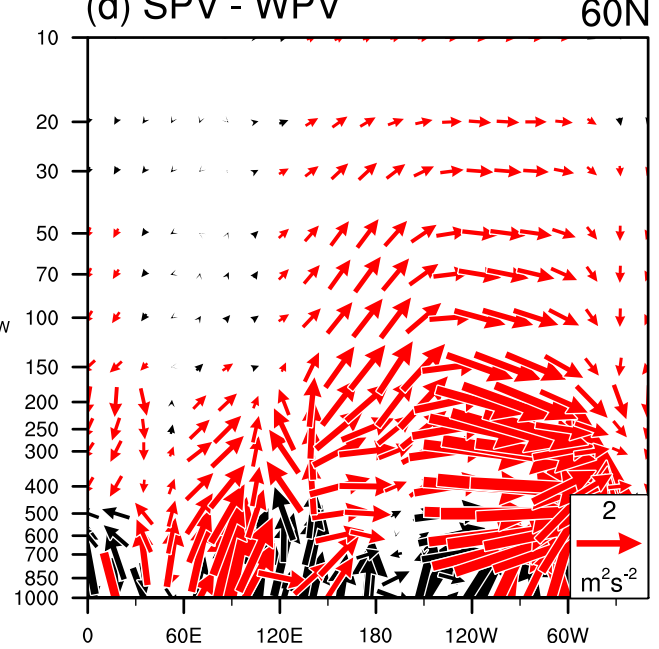

FIG. 6. Climatological distribution of the northern winter planetary wave activity fluxes at $100 \mathrm{hPa}$ in (a) SPV (b) WPV, and (c) the differences between SPV and WPV. Solid (dashed) contours indicate the upward (downward) components with an interval of $6 \times 10^{-5} \mathrm{~m}^{2} \mathrm{~s}^{-2}$ in (a) and (b) and $2 \times 10^{-5} \mathrm{~m}^{2} \mathrm{~s}^{-2}$ in (c). The shading and red arrows in (c) indicate the significance level at $5 \%$ based on a two-sided Student's $t$ test. (d) The differences in wave activity fluxes along $65^{\circ} \mathrm{N}$ between SPV and WPV. The vertical component is multiplied by $1 \times 10^{4}$. The red arrows in (d) indicate the significance level at $5 \%$ based on a two-sided Student's $t$ test.

vertical fluxes are weaker in WPV than in SPV models (Fig. 6b). The differences of wave activity fluxes between SPV and WPV models (Fig. 6c) reveal that there are strong upward wave activity flux anomalies over the North Pacific and significant downward planetary wave activity flux anomalies over the North Atlantic and northern North America in the upper troposphere and lower stratosphere. These fluxes combined with the significant eastward horizontal wave activity fluxes over North America and North Atlantic implies that there are significantly stronger waves reflected by the stratospheric PV in SPV models than in WPV models. Figure 6d further shows the differences of wave activity fluxes along $65^{\circ} \mathrm{N}$ between SPV and WPV models. There are apparent upward and eastward wave flux anomalies near $180^{\circ}$ from the upper troposphere to the stratosphere, and then they are reflected downward to troposphere around $30^{\circ} \mathrm{W}$. This result further supports our hypothesis that there are stronger wave reflections from the North Pacific to North Atlantic in the SPV models than in WPV models. The stronger wave reflection could help to strengthen the coupling of atmospheric circulation over the North Pacific and North Atlantic. Given stronger stratospheric PV events in the SPV models, this 
SPV

(a) Z200 \& WAF

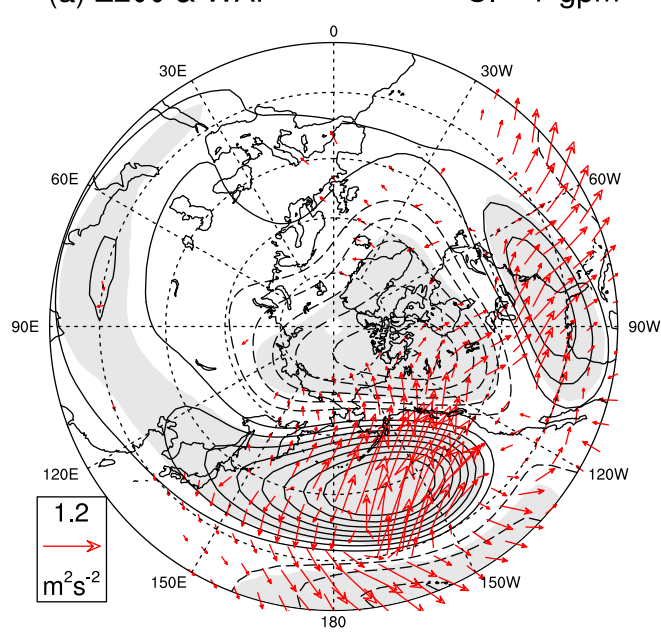

WPV

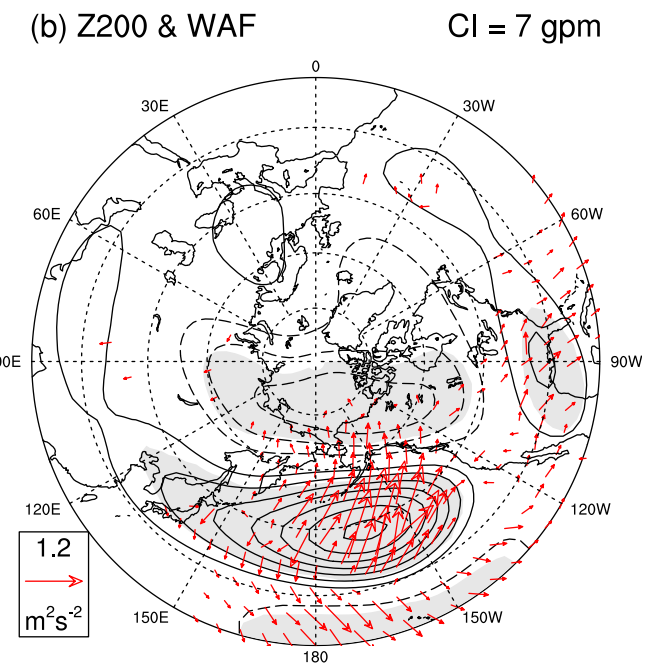

FIG. 7. (a) Wintertime 200-hPa geopotential height and wave activity flux anomalies regressed onto the normalized North Pacific index in SPV models. (b) As in (a), but for WPV models. The shading and arrows indicate the significance level at $5 \%$ based on a two-sided Student's $t$ test.

tight coupling between the North Pacific and North Atlantic is more frequent and induces a stronger correlation of atmospheric circulation between the North Pacific and North Atlantic on the interannual time scale.

To further delineate the dynamic processes that the stratospheric PV influences the North Pacific-North Atlantic coupling in more detail, we present in Fig. 7 the anomalies of 200-hPa geopotential height and wave activity flux anomalies associated with the North Pacific index in SPV and WPV models. In the SPV models, a clear Pacific-North America (PNA)-like wave train emanates from the anticyclonic anomalies in the North Pacific and the wave activity fluxes propagate more eastward to the North Atlantic, which generates a positive NAO-like disturbance over the North Atlantic and thus projects onto the positive AO-like pattern in the whole extratropical Northern Hemisphere (Fig. 7a). This result is similar to that based on the AL-IL index when it is in its active phases shown in Honda and Nakamura (2001). In this case, some of North Pacific variability is compounded in $\mathrm{AO}$ variability and thus induces a strong North Pacific center of AO pattern in SPV models (Fig. 4c). This result further supports the previous finding in Gong et al. (2017); that is, the stronger Pacific center in models results from the excessive relationships between the AO and NPM in most models. In contrast, the PNA-like wave train is weaker in the WPV models corresponding to less wave activity fluxes propagating from the North Pacific to the North Atlantic (Fig. 7b). In this case, the North Pacific-North Atlantic coupling is weaker. Therefore, there is less
North Pacific variability in the AO variability with a relatively weaker North Pacific center of the AO pattern in WPV models (Fig. 4d).

The above results suggest that there are more wave reflections from the North Pacific to the North Atlantic and more wave activity fluxes propagating from the North Pacific to the North Atlantic in models with stronger climatological stratospheric PV than that with weaker climatological stratospheric PV. As such, the frequency of strong North Pacific-North Atlantic coupling events is higher in SPV models than in WPV models. This strengthens the coupling of atmospheric circulation between the North Pacific and North Atlantic in SPV models in a statistical sense, and it is reflected in the stronger correlation of atmospheric circulation between the North Pacific and North Atlantic on an interannual time scale.

\section{Summary and discussion}

In this study, the sources of the diversity of the wintertime AO pattern especially for the North Pacific and North Atlantic centers is investigated in 27 CMIP5 models in the period 1961-2005. In the reanalysis, the North Pacific center of wintertime AO pattern is very weak, and the AO pattern resembles the NAO. Most of the CMIP5 models tend to simulate a stronger North Pacific center and a weaker North Atlantic center of the AO pattern than in reanalysis. The weak North Atlantic center of the AO pattern agrees with generally weak wintertime SLP variability over the North Atlantic in most of the models. 
(a) SPV - WPV

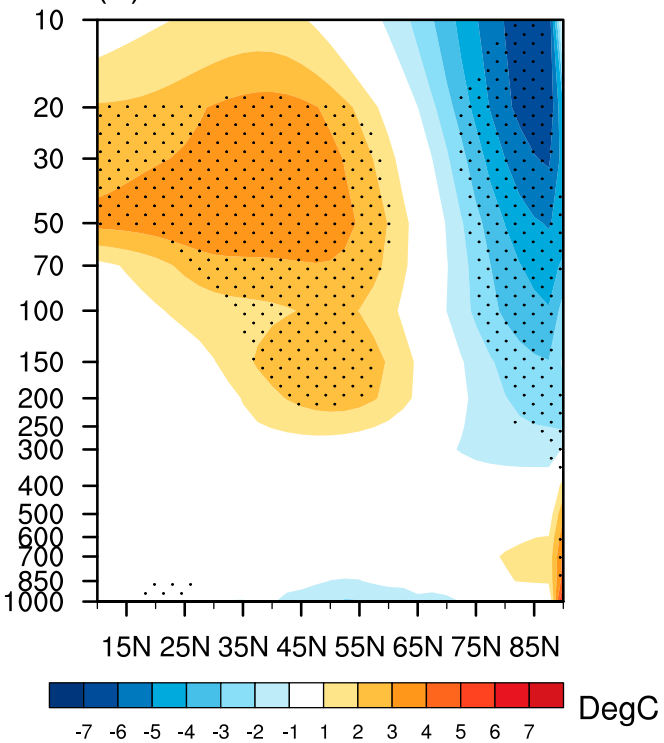

(b) SPV - JRA55

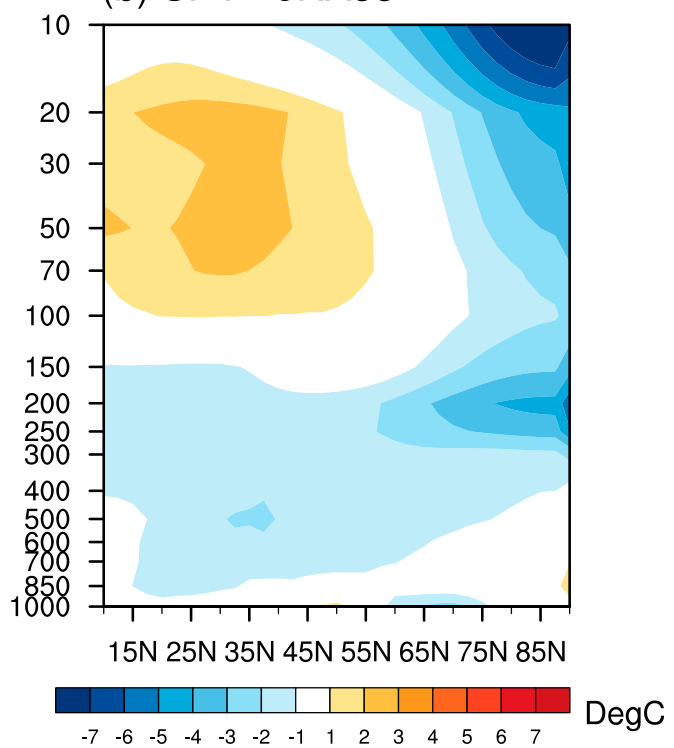

FIG. 8. (a) The differences of winter-mean (DJF) zonal-mean temperature for the period 1961-2005 between SPV and WPV. (b) As in (a), but for the differences between SPV and reanalysis. Dots in (a) indicate the significance level at $5 \%$ based on a two-sided Student's $t$ test.

The strength of the North Pacific-North Atlantic coupling is suggested to be an essential factor influencing the intensity of the North Pacific center in the AO pattern in models. Moreover, it is found that the North Pacific-North Atlantic coupling is related to the strength of the climatological stratospheric PV in CMIP5 models. That is, the climatological stratospheric $\mathrm{PV}$ is very likely to modulate the coupling between the North Pacific and North Atlantic variations in models. A comparative analysis is conducted by classifying the 27 models into two groups (SPV and WPV) according to the strength of the climatological stratospheric PV. It is found that the North Pacific center of the AO pattern is much stronger in SPV models than in WPV models. According to the wave flux activity differences between SPV and WPV models, there is visible wave reflection in SPV models, with upward and eastward wave flux anomalies over the North Pacific from the upper troposphere to the stratosphere. The waves are then reflected down to the North Atlantic. Thus, more wave activity fluxes propagate from the North Pacific to the North Atlantic in SPV models than in WPV models, leading to a strong coupling between the North Pacific and North Atlantic variations in SPV models. The North Pacific variability projects onto an AO-like pattern in the whole extratropical Northern Hemisphere. In this case, the AO is compounded with some of North Pacific variability in SPV models. Thus, this facilitates a stronger North Pacific center of the AO pattern. The strength of climatological stratospheric PV reflects the frequency of strong
North Pacific-North Atlantic coupling events in models (Fig. 5). Thus, the modulation of climatological stratospheric PV on the interannual North Pacific-North Atlantic coupling is statistically more prominent in SPV models than in WPV models in a statistical sense.

It is noted that the strength of stratospheric PV is tightly related to the stratospheric temperature (Osprey et al. 2013; Rao et al. 2015). The zonal-mean air temperature differences between SPV and WPV exhibit a clear dipole in the upper troposphere to the stratosphere, with cold and warm anomalies located north and south of $65^{\circ} \mathrm{N}$, respectively, strengthening the temperature and pressure gradient and causes stronger stratospheric PV in SPV (Fig. 8a). Furthermore, the strength of climatological-mean stratospheric PV is also significantly larger in SPV models than in reanalysis $\left(23 \mathrm{~m} \mathrm{~s}^{-1}\right.$ in SPV and $19.5 \mathrm{~m} \mathrm{~s}^{-1}$ in reanalysis). And the stratospheric polar temperature also shows an apparent cold bias in SPV models compared with reanalysis (Fig. 8b).

Besides, the climate models with higher vertical levels in its atmospheric component show better performances in simulating the North Pacific center of the AO pattern. Figure 9 shows the scatter diagram between the number of vertical levels and the amplitude of the North Pacific center of AO (NPCI) among models. A lower vertical resolution tends to correspond to a stronger North $\mathrm{Pa}$ cific center of $\mathrm{AO}$ pattern in models. The intermodel correlation coefficient between vertical levels and NPCI reaches -0.59 , exceeding the significance level at $1 \%$. 

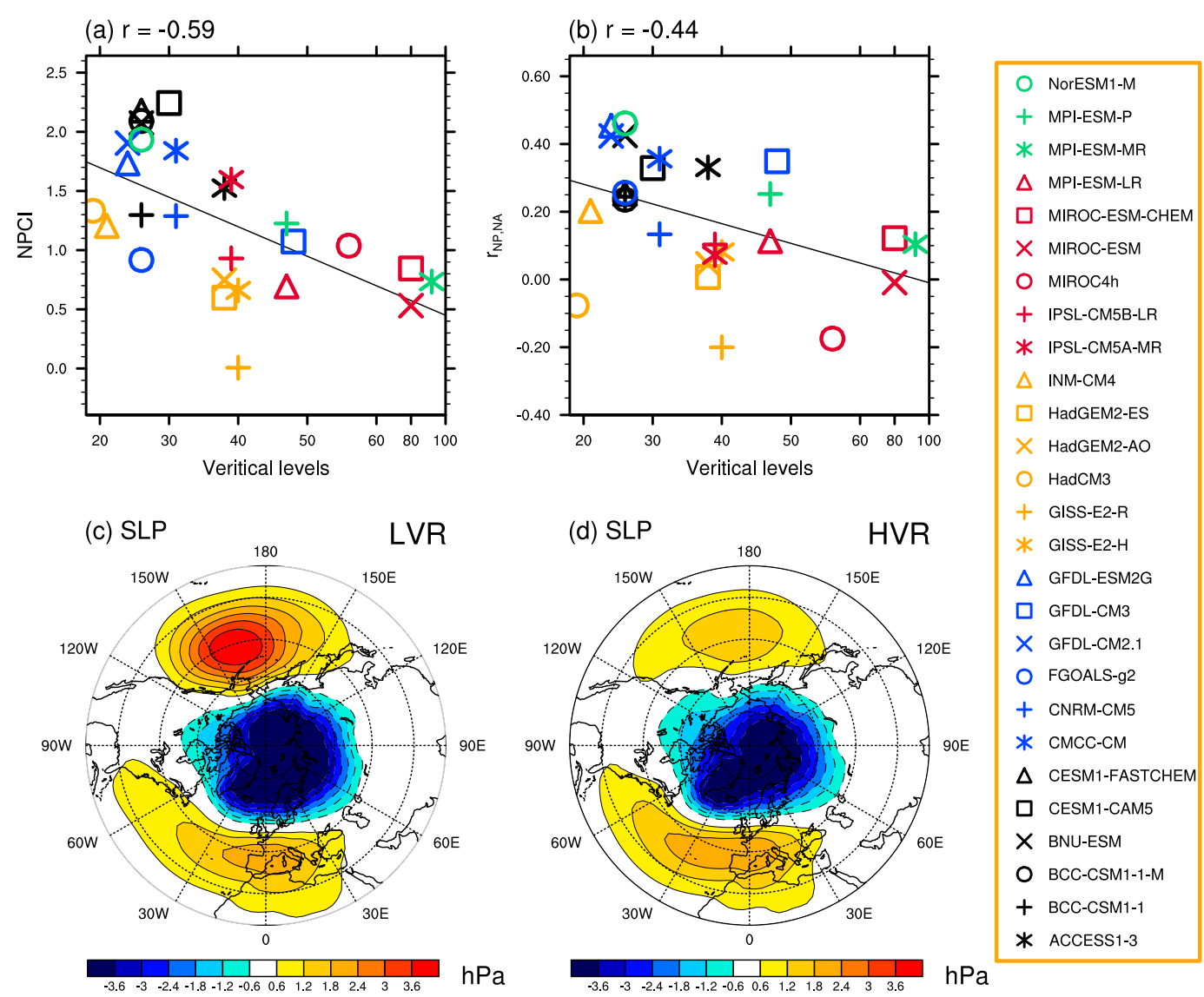

FIG. 9. (a) Scatterplot of the NPCI ( $y$ axis) vs number of vertical levels ( $x$ axis) in 27 CMIP5 models. (b) As in (a), but for the scatterplot of the $r_{\mathrm{NP}, \mathrm{NA}}$ ( $y$ axis) vs number of vertical levels ( $x$ axis). (c) The AO pattern of MME in the 10 lowest vertical resolution models (LVR, with less than 27 vertical levels). (d) As in (c), but for nine highest vertical resolution models (HVR, with more than 39 vertical levels).

Similarly, a lower vertical resolution tends to correspond to a stronger coupling between the North Pacific and North Atlantic centers in models (Fig. 9b). The intermodel correlation coefficient between vertical levels and $r_{\mathrm{NP}, \mathrm{NA}}$ reaches -0.44 , exceeding the significance level at $5 \%$. Based on the number of vertical levels, the AO pattern of MME in the 10 lowest vertical resolution models (LVR; the vertical levels less than 27) and 9 highest vertical resolution models (HVR; the vertical levels more than 39) are shown in Figs. 9c and 9d. The North Pacific center of the AO pattern is much stronger in LVR models than in HVR models.

Meanwhile, the intensity of the North Atlantic center is comparable in the two groups (Figs. 9c,d). These results suggest that the amplitude of the North Pacific center of the AO pattern is indeed associated with the vertical resolution of models. The models with higher vertical resolution show a better performance in reproducing the observed North Pacific center of AO than that the models with low vertical resolution. At the same time, there are significant differences of climatological zonal-mean zonal winds between the LVR and HVR models (Figs. 10a,b). The stratospheric polar night jet stream is much stronger in the LVR models than in the HVR models (Fig. 10c). This result suggests that the bias of strong stratospheric circulation in the models may result from the low vertical resolution and the inadequate description of the stratospheric processes in the models. These results support the previous findings that the stratospheric dynamical variability and related wave reflection are closely tied to the vertical resolution of CMIP5 models (Charlton-Perez et al. 2013; Shaw et al. 2014). The excessive stronger stratospheric PV in LVR models induces more planetary wave reflection from the North Pacific to the North Atlantic, which strengthens the coupling of atmospheric circulation between the North Pacific and the North Atlantic and results in a stronger North Pacific center in LVR models. The above results further suggest that increase in vertical resolution may improve the simulation of stratospheric 
(a) LVR

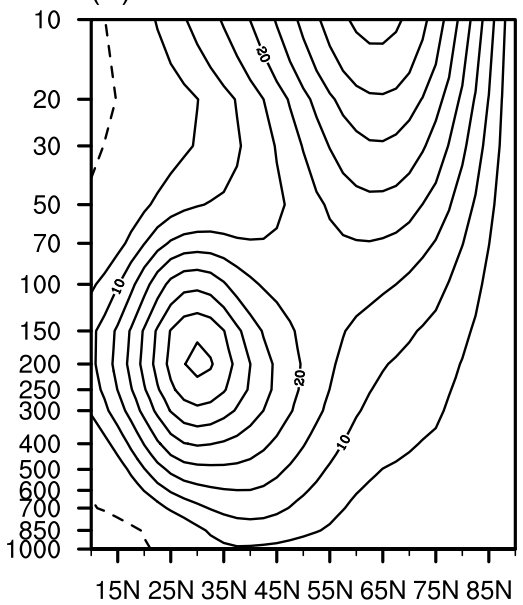

(b) HVR

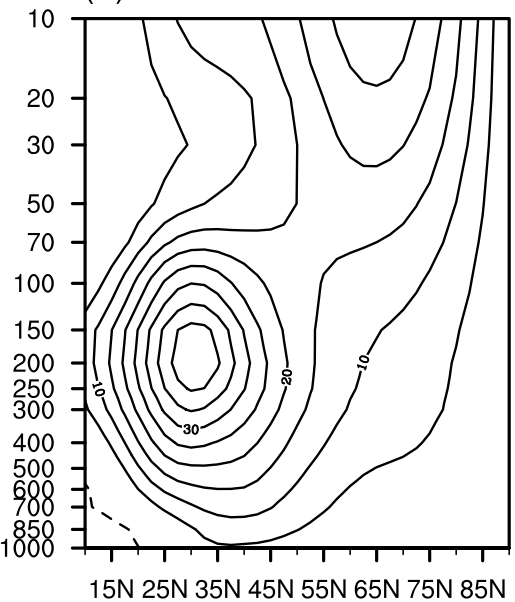

(c) LVR - HVR

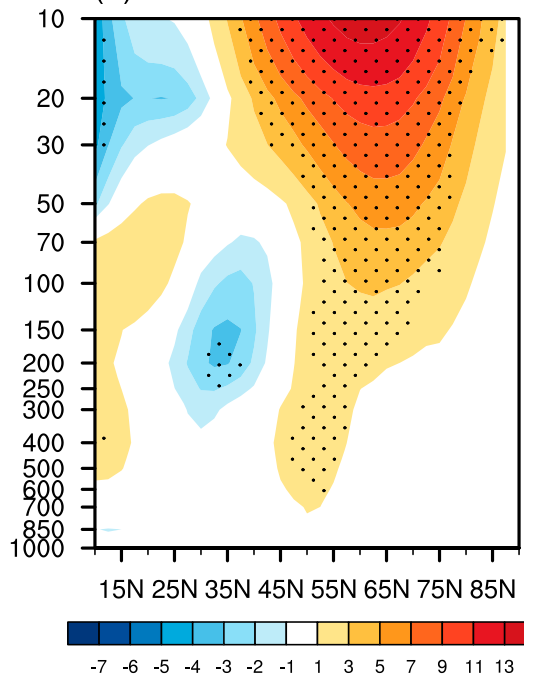

FIG. 10. Climatology of winter-mean (DJF) zonal-mean zonal wind in (a) LVR, (b) HVR, and (c) the differences between LVR and HVR. Contour intervals are $5 \mathrm{~m} \mathrm{~s}^{-1}$ in (a) and (b). Dots in (c) indicate the significance level at $5 \%$ based on a two-sided Student's $t$ test.

PV and thereby reduce the models' biases in the North Pacific-North Atlantic coupling and the amplitude of the North Pacific center of the AO pattern in models.

This study suggests that the magnitude of the North Atlantic center of the AO pattern is closely tied to the amplitudes of wintertime SLP variability over the North Atlantic in models. This intermodel relationship is also found in the Arctic center of AO pattern. When the model has a larger SLP variability in the Arctic region, the Arctic center is also larger (not shown). However, it is unclear what mechanisms are responsible for the diversity intensity of SLP variability among models. It should be noted that in most of WPV models, although the climatological stratospheric PV in WPV is weaker than that in reanalysis, the North Pacific-North Atlantic coupling and the amplitudes of the North Pacific center of the AO pattern are still somewhat stronger than in reanalysis. This bias may be related to the systematic bias of model simulation and needs to be investigated further in the future. Last but not least, some CMIP5 models were excluded from the present analysis because the AO is not the leading mode in these models. The PNA-like pattern is the first EOF mode in those models when the wintertime SLP over the Northern Hemisphere is analyzed (not shown). The reason for this altered sequence of EOF modes is unclear and deserves further investigation in the future.

Acknowledgments. We thank the three anonymous reviewers and the editor Darryn Waugh for their careful reviews and constructive suggestions that help to improve the manuscript substantially. We acknowledge the climate modeling groups listed in Table 1 for making available their model output, and the Working Group on Coupled Modeling (WGCM) of the World Climate Research Program (WCRP) for coordinating the CMIP5 project. This work was supported by the National Key Research and Development Program of China (2018YFC1506003) and the National Natural Science Foundation of China (41721004, 41605060).

\section{REFERENCES}

Ambaum, M. H. P., B. J. Hoskins, and D. B. Stephenson, 2001: Arctic Oscillation or North Atlantic Oscillation? J. Climate, 14, 3495-3507, https://doi.org/10.1175/1520-0442(2001)014<3495: AOONAO $>2.0 . \mathrm{CO} ; 2$.

Castanheira, J. M., and H. F. Graf, 2003: North Pacific-North Atlantic relationships under stratospheric control? J. Geophys. Res., 108, 4036, https://doi.org/10.1029/2002JD002754.

Cattiaux, J., and C. Cassou, 2013: Opposite CMIP3/CMIP5 trends in the wintertime northern annular mode explained by combined local sea ice and remote tropical influences. Geophys. Res. Lett., 40, 3682-3687, https://doi.org/10.1002/grl.50643.

Charlton-Perez, A., and Coauthors, 2013: On the lack of stratospheric dynamical variability in low-top versions of the CMIP5 models. J. Geophys. Res., 118, 2494-2505, https://doi.org/ $10.1002 /$ jgrd.50125.

Charney, J. G., and P. G. Drazin, 1961: Propagation of planetaryscale disturbances from the lower into the upper atmosphere. J. Geophys. Res., 66, 83-109, https://doi.org/10.1029/ JZ066i001p00083.

Chen, W., H. Graf, and M. Takahashi, 2002: Observed interannual oscillation of planetary wave forcing in the Northern Hemisphere winter. Geophys. Res. Lett., 29, 2073, https://doi.org/ 10.1029/2002GL016062.

, M. Takahashi, and H. Graf, 2003: Interannual variations of stationary planetary wave activity in the northern winter 
troposphere and stratosphere and their relations to NAM and SST. J. Geophys. Res., 108, 4797, https://doi.org/10.1029/ 2003JD003834.

- X. Lan, L. Wang, and Y. Ma, 2013: The combined effects of the ENSO and the Arctic Oscillation on the winter climate anomalies in East Asia. Chin. Sci. Bull., 58, 1355-1362, https:// doi.org/10.1007/s11434-012-5654-5.

Deser, C., 2000: On the teleconnectivity of the "Arctic Oscillation.” Geophys. Res. Lett., 27, 779-782, https://doi.org/10.1029/ 1999GL010945.

Dommenget, D., and M. Latif, 2002: A cautionary note on the interpretation of EOF. J. Climate, 15, 216-225, https://doi.org/ 10.1175/1520-0442(2002)015<0216:ACNOTI >2.0.CO;2.

Feldstein, S. B., and C. Franzke, 2006: Are the North Atlantic Oscillation and the northern annular mode distinguishable? J. Atmos. Sci., 63, 2915-2930, https://doi.org/10.1175/JAS3798.1.

Gong, H., L. Wang, W. Chen, R. Wu, K. Wei, and X. Cui, 2014: The climatology and interannual variability of the East Asian winter monsoon in CMIP5 models. J. Climate, 27, 1659-1678, https://doi.org/10.1175/JCLI-D-13-00039.1.

,,,--- D. Nath, G. Huang, and W. Tao, 2015: Diverse influences of ENSO on the East Asian-western Pacific winter climate tied to different ENSO properties in CMIP5 models. J. Climate, 28, 2187-2202, https://doi.org/10.1175/JCLI-D-1400405.1.

,,--- X. Chen, and D. Nath, 2017: Biases of the wintertime Arctic Oscillation in CMIP5 models. Environ. Res. Lett., 12, 014001, https://doi.org/10.1088/1748-9326/12/1/014001.

,,--- , and D. Nath, 2018: Multidecadal fluctuation of the wintertime Arctic Oscillation pattern and its implication. J. Climate, 31, 5595-5608, https://doi.org/10.1175/JCLI-D-17-0530.1.

Harada, Y., and Coauthors, 2016: The JRA-55 Reanalysis: Representation of atmospheric circulation and climate variability. J. Meteor. Soc. Japan, 94, 269-302, https://doi.org/10.2151/ jmsj.2016-015.

Honda, M., and H. Nakamura, 2001: Interannual seesaw between the Aleutian and Icelandic lows. Part II: Its significance in the interannual variability over the wintertime Northern Hemisphere. J. Climate, 14, 4512-4529, https://doi.org/10.1175/15200442(2001)014<4512:ISBTAA $>2.0$. CO;2.

Hurrell, J. W., 1995: Decadal trends in the North Atlantic oscillation: Regional temperatures and precipitation. Science, 269, 676-679, https://doi.org/10.1126/science.269.5224.676.

Itoh, H., 2008: Reconsideration of the true versus apparent Arctic Oscillation. J. Climate, 21, 2047-2062, https://doi.org/10.1175/ 2007JCLI2167.1.

Kobayashi, S., and Coauthors, 2015: The JRA-55 Reanalysis: General specifications and basic characteristics. J. Meteor. Soc. Japan, 93, 5-48, https://doi.org/10.2151/jmsj.2015-001.

Miller, R. L., G. A. Schmidt, and D. T. Shindell, 2006: Forced annular variations in the 20th century Intergovernmental Panel on Climate Change Fourth Assessment Report models. J. Geophys. Res., 111, D18101, https://doi.org/10.1029/ 2005JD006323.

Osprey, S. M., L. J. Gray, S. C. Hardiman, N. Butchart, and T. J. Hinton, 2013: Stratospheric variability in twentieth-century CMIP5 simulations of the Met Office climate model: High top versus low top. J. Climate, 26, 1595-1606, https://doi.org/ 10.1175/JCLI-D-12-00147.1.
Perlwitz, J., and H.-F. Graf, 2001: Troposphere-stratosphere dynamic coupling under strong and weak polar vortex conditions. Geophys. Res. Lett., 28, 271-274, https://doi.org/10.1029/ 2000GL012405.

Plumb, R. A., 1985: On the three-dimensional propagation of planetary waves. J. Atmos. Sci., 42, 217-229, https://doi.org/ 10.1175/1520-0469(1985)042<0217:OTTDPO > 2.0.CO;2.

Rao, J., R. Ren, and Y. Yang, 2015: Parallel comparison of the northern winter stratospheric circulation in reanalysis and in CMIP5 models. Adv. Atmos. Sci., 32, 952-966, https://doi.org/ 10.1007/s00376-014-4192-2.

Rogers, J., and M. McHugh, 2002: On the separability of the North Atlantic Oscillation and Arctic Oscillation. Climate Dyn., 19, 599-608, https://doi.org/10.1007/s00382-002-0247-7.

Shaw, T., J. Perlwitz, and O. Weiner, 2014: Tropospherestratosphere coupling: Links to North Atlantic weather and climate, including their representation in CMIP5 models. J. Geophys. Res., 119, 5864-5880, https://doi.org/10.1002/ 2013JC009598.

Stoner, A. M. K., K. Hayhoe, and D. J. Wuebbles, 2009: Assessing general circulation model simulations of atmospheric teleconnection patterns. J. Climate, 22, 4348-4372, https://doi.org/ 10.1175/2009JCLI2577.1.

Sun, J., and B. Tan, 2013: Mechanism of the wintertime Aleutian low-Icelandic low seesaw. Geophys. Res. Lett., 40, 4103-4108, https://doi.org/10.1002/grl.50770.

Tan, B., L. Suo, and J. Huang, 2008: Variability of the coupling between surface air temperature and northern annular mode at various levels. Acta Meteor. Sin., 22, 277-283.

Thompson, D. W. J., and J. M. Wallace, 1998: The Arctic Oscillation signature in the wintertime geopotential height and temperature fields. Geophys. Res. Lett., 25, 1297-1300, https:// doi.org/10.1029/98GL00950.

—, and - 2000: Annular modes in the extratropical circulation. Part I: Month-to-month variability. J. Climate, 13, 1000-1016, https://doi.org/10.1175/1520-0442(2000)013<1000: AMITEC $>2.0 . \mathrm{CO} ; 2$.

,-- , and G. C. Hegerl, 2000: Annular modes in the extratropical circulation. Part II: Trends. J. Climate, 13, 1018-1036, https://doi.org/10.1175/1520-0442(2000)013<1018: AMITEC $>2.0 . \mathrm{CO} ; 2$.

Wallace, J. M., 2000: North Atlantic Oscillation/annular mode: Two paradigms - one phenomenon. Quart. J. Roy. Meteor. Soc., 126, 791-805, https://doi.org/10.1256/smsqj.56401.

— and D. W. J. Thompson, 2002: The Pacific center of action of the Northern Hemisphere annular mode: Real or artifact? J. Climate, 15, 1987-1991, https://doi.org/10.1175/15200442(2002)015<1987:TPCOAO > 2.0.CO;2.

Wang, L., and W. Chen, 2010: Downward Arctic Oscillation signal associated with moderate weak stratospheric polar vortex and the cold December 2009. Geophys. Res. Lett., 37, L09707, https://doi.org/10.1029/2010GL042659.

Yuan, J., W. Li, R. E. Kopp, and Y. Deng, 2018: Response of subtropical stationary waves and hydrological extremes to climate warming in boreal summer. J. Climate, 31, 10165 10 180, https://doi.org/10.1175/JCLI-D-17-0401.1.

Zuo, J. Q., W. J. Li, and H. L. Ren, 2013: Representation of the Arctic Oscillation in the CMIP5 models. Adv. Climate Change Res., 4, 242-249, https://doi.org/10.3724/SP.J.1248.2013.242. 